\title{
Energy-efficient Joint Power Allocation and Channel Selection for D2D Communications
}

\author{
Guifang $\mathrm{Ma}^{\dagger}$ \\ State Key Laboratory of \\ Alternate Electrical Power \\ System with Renewable \\ Energy Sources \\ School of Electrical and \\ Electronic Engineering, North \\ China Electric Power \\ University \\ 102206, Beijing, China \\ mgfxiamu@163.com
}

\author{
Zhenyu Zhou \\ State Key Laboratory of \\ Alternate Electrical Power \\ System with Renewable \\ Energy Sources \\ School of Electrical and \\ Electronic Engineering, North \\ China Electric Power \\ University \\ 102206, Beijing, China \\ zhenyu_zhou@ncepu.edu.cn 835697648@qq.com
}

\begin{abstract}
Device-to-device (D2D) communications have gained great attentions due to the potential and numerous benefits for cellular networks. However, it also brings tremendous resource allocation challenges for the sake of the constraint of battery life. Up to now, there are limited works attempt to prolong the battery life by improving the energy efficiency (EE). In this paper, we study how to perform resource allocation to increase $\mathrm{EE}$ in a interference limited environment under a noncooperative game model. Each D2D pair can reuse all or part of the channel resources allocated to cellular users. An energy-efficient joint power allocation and channel selection is proposed by employing the nonlinear fractional programming. We obtain the optimal power allocation and channel selection through an iterative algorithm called Dinkelbach method. Finally, the algorithm proposed in this paper is verified by simulation.
\end{abstract}

\section{Keywords}

Energy-efficiency, D2D, resource allocation, game theory, Dinkelbach method

\section{INTRODUCTION}

Device-to-device(D2D) communications enable user equipments (UE) in close proximity to communicate with each

\footnotetext{
* This work was partially supported by the Fundamental Research Funds for the Central Universities under Grant Number 13MS19, 14MS11, China Mobile Communication Co. Ltd. Research Institute (CMRI), and China Electric Power Research Institute (CEPRI) of State Grid Corporation of China (SGCC)

${ }^{\dagger}$ Miss. Ma insisted her name be first.
}

other directly without extra hops through the central base station(BS) [1]. D2D communication has great potential to improve the spectral efficiency (SE) and energy efficiency (EE) due to the proximity gain, spectrum reuse gain and hop gain [2]. However, there are paramount challenges needed to be addressed to harvest these potential benefits considering battery constraints. There are limited works discussing how to perform resource allocation to increase EE using different algorithms.

An iterative combinatorial auction algorithm was proposed in [3], which employs the Peukertar's law to model the battery lifetime. A novel device beaconing scheme facilitating device discovery in a larger network was studied in [4], where the EE was used to evaluate the proposed mechanism. Another energy-efficient resource allocation scheme was proposed in [5], which minimizes the total transmit power levels for D2D pairs and cellular UEs. How mobility impacts EE in a general framework of an LTE-Advanced network was investigated in [6]. In [7], two novel resource allocation schemes were proposed to increase the throughput and reduce the overall energy cost by mitigating interference in the cell level and scheduling resources in the user level in an energy-efficient way. The optimal power allocation based on three different modes to maximize the EE was presented in [8], and close analytical approximations based on Taylorseries expansion were devived. In [9], D2D discovery mechanisms discussed in the 3GPP were studied in terms of energy consumption aspects by optimizing network resource utilization. Two energy-aware game theoretic medium access control (MAC) strategies were proposed in [10], in which the D2D users were respectively under distributed and networkassisted scenarios. The trade-off between SE and EE was analyzed in [11] assuming that all the channel are available to each user. [12] proposed an iterative optimization algorithm to maximize $\mathrm{EE}$ of each user without considering channel selection.

However, the above mentioned works have not directly optimized the energy-efficiency, which is defined as the ratio of $\mathrm{SE}$ and total power. The joint power allocation and channel selection problem is a complex combinatorial problem and 
there is no closed-form solution. In this paper, the properties of non linear fractional programming are employed in the resource allocation under a non-cooperative game to transform the non-convex problem to a convex problem. Moreover, the channel selection indicator is relaxed to be a real value instead of a Boolean variable to avoid the problem becoming a integer programming problem. Then we propose an iterative algorithm based on a Dinkelbach method subject to the QoS provisioning and transmission power constraints. Finally, the effectiveness of the proposed algorithm is verified by simulation.

This paper is organized as follows. The section 2 presents system model and analyzes different kinds of mutual interference among D2D UEs and cellular UEs. The section 3 introduces the distributed iterative optimization algorithm to optimize energy-efficiency.Simulation results and analysis are presented. Finally, the main conclusions of our work are summarized.

\section{SYSTEM MODEL AND PROBLEM FOR- MULATION}

\subsection{System Model}

In this paper, D2D communications are considered underlaying a single cellular network. We allow D2D UEs to reuse the channel resources allocated to cellular UEs to maximize SE. As illustrated in Fig. 1, cellular UEs establish direct link to BS to communicate, while D2D UEs communicate with each other directly. There is no interference among cellular UEs for the orthogonal channel. Each cellular UEs is allocated to multiple subchannels, which D2D UEs determine to reuse entirely or partially. In this reusing mode, there are different interference scenarios in uplink communication. When D2D pairs reuse uplink cellular resources, the interferences for $\mathrm{D} 2 \mathrm{D}$ receivers $\left(R_{x}\right.$ of $\mathrm{D}_{2} \mathrm{D}_{1}$ or $\left.\mathrm{D}_{2} \mathrm{D}_{2}\right)$ come from co-channel cellular UEs (cellular UE1 and cellular UE2) and other co-channel D2D transmitters in the same cell $\left(T_{x}\right)$. The interferences subject to BS come from all the D2D UEs reusing the same channel.

We focus on the distributed resource allocation problem modeled as a noncooperative game, in which each UE maximize its own EE regardless of others. Before formulating our problem, there are some key parameters needed to to be defined. We assume that the number of D2D UEs and cellular UEs are $N$ and $K$ respectively. Each cellular UEs has $M$ available channels, so the total number of channels D2D UEs can reuse is denoted as $L=M K$.Since D2D UEs may not reuse all of the channels allocated to cellular UEs, the selection indicator $s_{i}^{l}$ [13]defined as follows:

$$
s_{i}^{l}= \begin{cases}1, & \text { if the } l \text {-th channel is used by } i \text {-th D2D pair; } \\ 0, & \text { otherwise }\end{cases}
$$

While in order to avoid the complex integer programming problem, $s_{i}^{l}$ is relaxed to be a real value between zero and one instead of a Boolean, i.e., $0 \leq s_{i}^{l} \leq 1$. For facilitating the algorithm, three new variables are defined:

$$
p_{i}^{l}=\tilde{p}_{i}^{l} s_{i}^{l}
$$

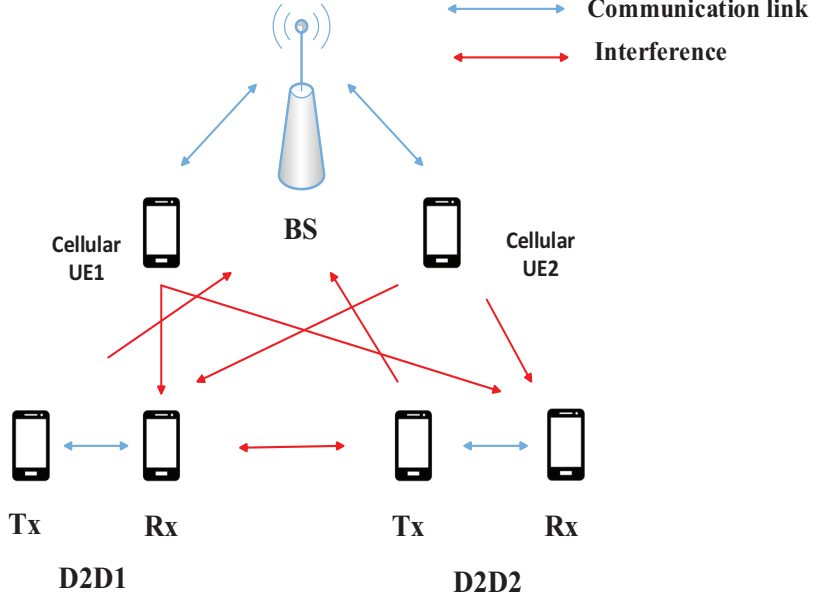

Figure 1: System model of D2D communications with uplink channel reuse.

$$
p_{j}^{l}=\tilde{p}_{j}^{l} s_{j}^{l}
$$

where $\tilde{p}_{i}^{l}, \tilde{p}_{k}^{l}, \tilde{p}_{j}^{l}$ are the transmission power of the $i$-th transmitter, the $k$-th cellular UE, and the $j$-th D2D transmitter in the $l$-th channel respectively. $p_{i}^{l}$, and $p_{j}^{l}$ are the actual selected transmission power of the $i$-th D2D transmitter, the $k$-th cellular UE, and the $j$-th D2D transmitter in the $l$-th channel respectively.

Then the signal to interference plus noise ratio (SINR) of the $i$-th D2D pair in the $l$-th channel is given by

$$
\gamma_{i}^{l}=\frac{p_{i}^{l} g_{i}^{l}}{\tilde{p}_{k}^{l} g_{k, i}^{l}+\sum_{j=1, j \neq i}^{N} p_{j}^{l} g_{j, i}^{l}+N_{0}},
$$

where $g_{i}^{l}$ is the channel gain of the $i$-th D2D pair, $g_{k, i}^{l}$ is the interference channel gain between the $k$-th cellular UE and the $i$-th D2D receiver, and $g_{j, i}^{l}$ is the interference channel gain between the $j$-th $\mathrm{D} 2 \mathrm{D}$ transmitter and the $i$-th D2D receiver. $N_{0}$ is the noise power. $p_{k}^{l} g_{k, i}^{l}$ and $\sum_{j=1, j \neq i}^{N} p_{j}^{l} g_{j, i}^{l}$ denote the interference from the cellular UE and the other D2D pairs that reuse the $l$-th channel respectively.

The received SINR of the $k$-th cellular UE at the BS in the $l$-th channel is given by

$$
\gamma_{k}^{l}=\frac{\tilde{p}_{k}^{l} g_{k}^{l}}{\sum_{i=1}^{N} p_{i}^{l} g_{i, k}^{l}+N_{0}}
$$

where $g_{k}^{l}$ is the channel gain between the $k$-th cellular UE and the BS, $g_{i, k}^{l}$ is the interference channel gain between the $i$-th D2D transmitter and the BS in the $l$-th channel. $\sum_{i=1}^{N} p_{i}^{l} g_{i, k}^{l}$ denotes the interference from all of the D2D pairs to the BS in the $l$-th channel.

The achievable rates of the $i$-th $\mathrm{D} 2 \mathrm{D}$ pair and the $k$-th cel- 
lular UE are given by

$$
\begin{aligned}
r_{i}^{l} & =\sum_{l=1}^{L} \log _{2}\left(1+\gamma_{i}^{l}\right), \\
r_{k}^{l} & =\sum_{l=k M+1}^{(k+1) M} \log _{2}\left(1+\gamma_{k}^{l}\right) .
\end{aligned}
$$

The total power consumption of the $i$-th D2D pair and the $k$-th cellular UE are given by

$$
\begin{aligned}
p_{i, \text { total }}^{l} & =\sum_{l=1}^{L} \frac{1}{\eta} p_{i}^{l}+2 p_{\text {cir }}, \\
\tilde{p}_{k, \text { total }}^{l} & =\sum_{l=k M+1}^{(k+1) M} \frac{1}{\eta} \tilde{p}_{k}^{l}+p_{\text {cir }},
\end{aligned}
$$

where $p_{i, \text { total }}^{l}$ is the total power consumption of the $i$-th D2D pair, which is composed of the transmission power over all of the $L$ channels, i.e., $\sum_{l=1}^{L} \frac{1}{\eta} p_{i}^{l}$, and the circuit power of both the D2D transmitter and receiver, i.e., $2 p_{\text {cir }}$. The circuit power of any UE is assumed as the same and is denoted as $p_{\text {cir }} . \eta$ is the power amplifier (PA) efficiency, i.e., $0<$ $\eta<1$. $\tilde{p}_{k, \text { total }}^{l}$ is the total power consumption of the $k$-th cellular UE, which is composed of the transmission power $\sum_{l=k M+1}^{(k+1) M} \frac{1}{\eta} \tilde{p}_{k}^{l}$ and the circuit power only at the transmitter side. The power consumption of the BS is not taken into consideration.

\subsection{Problem Formulation}

In this section, we formulate the EE maximization problem of $i$-th D2D pair and $k$-th cellular respectively under a noncooperative game. In the game, each UE wants to maximize its own EE regardless of others. The strategies set of the $l$-th D2D transmitter and $k$-th cellular UEs are denoted as $p_{i}^{l}$ and $p_{k}^{l}$ respectively. For the $i$-th D2D pair, its EE $U_{i, E E}^{d}$ depends not only on $p_{i}^{l}$, but also on the strategies taken by other UEs. $U_{i, E E}^{l}$ is defined as

$$
\begin{aligned}
& U_{i, E E}^{l}\left(p_{i}^{l}\right) \\
& =\frac{r_{i}^{l}}{p_{i, \text { total }}^{l}}=\frac{\sum_{l=1}^{L} \log _{2}\left(1+\frac{p_{i}^{l} g_{i}^{l}}{\tilde{p}_{k}^{l} \tilde{g}_{k, i}^{l}+\sum_{j=1, j \neq i}^{N} p_{j}^{l} g_{j, i}^{l}+N_{0}}\right)}{\sum_{l=1}^{L} \frac{1}{\eta} p_{i}^{l}+2 p_{c i r}} .
\end{aligned}
$$

Therefore, the EE maximization problem of the $i$-th D2D pair is formulated as

$$
\begin{aligned}
& \max . \quad U_{i, E E}^{l}\left(p_{i}^{l}\right) \\
& \text { s.t. } \quad C 1, C 2 . \\
& C 1: r_{i}^{l} \geq R_{i, \min }^{l}, \\
& C 2: 0 \leq \sum_{l=1}^{L} p_{i}^{l} \leq p_{i, \max }^{l} .
\end{aligned}
$$

Similarly, the EE of the $k$-th cellular UE $U_{k, E E}^{c}$ is defined as

$$
U_{k, E E}^{l}\left(\tilde{p}_{k}^{l}\right)=\frac{r_{k}^{l}}{\tilde{p}_{k, \text { total }}^{l}}=\frac{\sum_{l=k M+1}^{(k+1) M} \log _{2}\left(1+\frac{\tilde{p}_{k}^{l} l_{k}^{l}}{\sum_{i=1}^{N} p_{i}^{l} g_{i, k}^{l}+N_{0}}\right)}{\sum_{l=k M+1}^{(k+1) M} \frac{1}{\eta} \tilde{p}_{k}^{l}+p_{\text {cir }}} .
$$

The corresponding EE maximization problem is formulated as

$$
\begin{gathered}
\max . \quad U_{k, E E}^{l}\left(\tilde{p}_{k}^{l}\right) \\
\text { s.t. } C 3, C 4 . \\
C 3: r_{k}^{l} \geq R_{k, \min }^{l}, \\
C 4: 0 \leq \sum_{l=k M+1}^{(k+1) M} \tilde{p}_{k}^{l} \leq \tilde{p}_{k, \text { max }}^{l} .
\end{gathered}
$$

The constraints $\mathrm{C} 1$ and $\mathrm{C} 3$ specify the QoS requirements in terms of minimum transmission rate. $\mathrm{C} 2$ and $\mathrm{C} 4$ are the non-negative constraints on the power allocation variables.

\section{THE ITERATIVE OPTIMIZATION AL- GORITHM}

As we know, the objective functions in (10) and (14) are both in fractional form, there is no closed-form solution for the non-convex problem. We can transform (10) and (14) into the subtractive form equivalent to the original problem by employing the nonlinear fractional programming. The transformed problem is a convex problem. The maximum EE of the $i$-th D2D pair $q_{i}^{l *}$ and the optimal solution of the $k$-th cellular UE $q_{k}^{l *}$ can be expressed as:

$$
\begin{aligned}
& q_{i}^{l *}=\max \cdot U_{i, E E}^{c}\left(p_{i}^{l},\right)=\frac{r_{i}^{l}\left(p_{i}^{l *}\right)}{p_{i, \text { total }}^{l}\left(p_{i}^{l *}\right)} . \\
& q_{k}^{l *}=\max \cdot U_{k, E E}^{l}\left(\tilde{p}_{k}^{l}\right)=\frac{r_{k}^{l}\left(\tilde{p}_{k}^{l *}\right)}{\tilde{p}_{k, \text { total }}^{l}\left(\tilde{p}_{k}^{l *}\right)} .
\end{aligned}
$$

According to [14], the following theorems can be proved, which shows the equivalence of the transformed problem. Theorem 1: The maximum EE $q_{i}^{l *}$ can be obtained if

$$
\max . r_{i}^{l}\left(p_{i}^{l}\right)-q_{i}^{l *} p_{i, \text { total }}^{l}\left(p_{i}^{l}\right)=r_{i}^{l}\left(p_{i}^{l *}\right)-q_{i}^{l *} p_{i, \text { total }}^{l}\left(p_{i}^{l *}\right)=0 \text {. }
$$

Theorem 2: The maximum EE $\tilde{q}_{k}^{l *}$ can be obtained if

$$
\max . r_{k}^{l}\left(\tilde{p}_{k}^{l}\right)-\tilde{q}_{k}^{l *} \tilde{p}_{k, \text { total }}^{l}\left(\tilde{p}_{k}^{l}\right)=r_{k}^{l}\left(\tilde{p}_{k}^{l *}\right)-\tilde{q}_{k}^{l *} \tilde{p}_{k, \text { total }}^{l}\left(\tilde{p}_{k}^{l *}\right)=0 .
$$

Thus unique optimal solutions can be achieved by solving the following transformed optimization problems:

$$
\begin{aligned}
& \max \cdot r_{i}^{l}\left(p_{i}^{l}\right)-q_{i}^{l} p_{i, \text { total }}^{l}\left(p_{i}^{l}\right) \\
& \text { s.t. } C 1, C 2 . \\
& \max \cdot r_{k}^{l}\left(\tilde{p}_{k}^{l}\right)-\tilde{q}_{k}^{l} \tilde{p}_{k, \text { total }}^{l}\left(\tilde{p}_{k}^{l}\right) \\
& \text { s.t. } C 3, C 4 .
\end{aligned}
$$

The Lagrangian function of the primal problem (23) can be given as

$$
\begin{aligned}
& \mathcal{L}_{E E}\left(p_{i}^{l}, \alpha_{i}, \beta_{i}\right)=r_{i}^{l}\left(p_{i}^{l}\right)-q_{i}^{l} p_{i, \text { total }}^{l}\left(p_{i}^{l}\right) \\
& +\alpha_{i}\left(r_{i}^{l}\left(p_{i}^{l}\right)-R_{i, \text { min }}^{l}\right)-\beta_{i}\left(\sum_{l=1}^{L} p_{i}^{l}-p_{i, \text { max }}^{l}\right),
\end{aligned}
$$

where $\alpha_{i}, \beta_{i}$ are the lagrange multipliers associated with different constraints. The dual problem can be expressed as

$$
\min _{\left(\alpha_{i} \geq 0, \beta_{i} \geq 0\right)} \quad \max _{\left(p_{i}^{l}\right)} \cdot \mathcal{L}_{E E}\left(p_{i}^{l}, \alpha_{i}, \beta_{i}\right)
$$




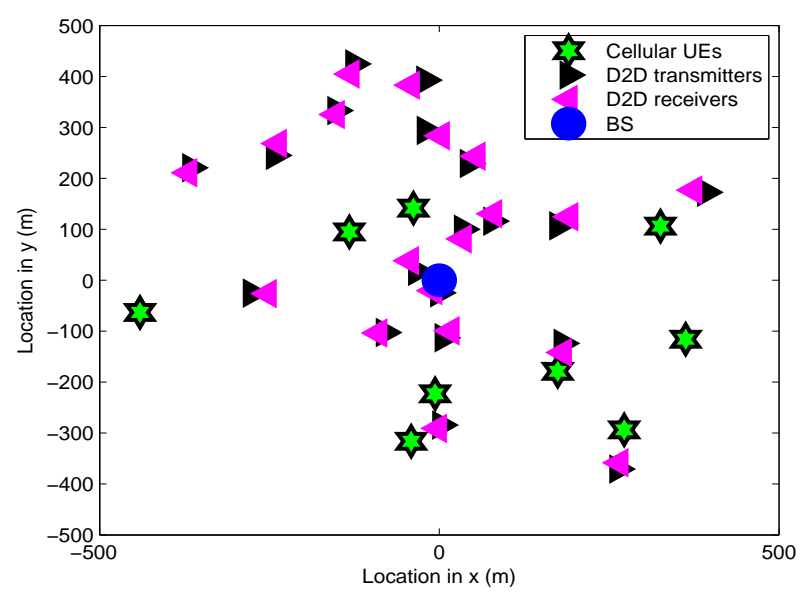

Figure 2: The locations of D2D UEs and cellular UEs generated in one simulation $(N=20, K=10$, the cell radius is $500 \mathrm{~m}$, and maximum D2D distance is $25 \mathrm{~m})$.

The dual problem can be decomposed into two layers, the inner maximization to solve the power allocation problem, the outer minimization to find the corresponding Lagrange multipliers. The dual problem can be solved by addressing both problems iteratively, where in each iteration, the Karush-Kuhn-Tucker (KKT) condition [15] is used to find the optimum solutions for a fixed set of Lagrange multipliers. For any given $q_{i}^{l}$ or $q_{k}^{l}$, the solution are given by

$$
\begin{gathered}
p_{i}^{l}=\left[\frac{\eta\left(1+\alpha_{i}\right) \log _{2} e}{q_{i}^{d}+\eta \beta_{i}}-\frac{\tilde{p}_{k}^{l} g_{k, i}^{l}+\sum_{j=1, j \neq i}^{N} p_{j}^{l} g_{j, i}^{l}+N_{0}}{g_{i}^{l}}\right]_{(26)}^{+}, \\
\tilde{p}_{k}^{l}=\left[\frac{\eta\left(1+\delta_{k}\right) \log _{2} e}{\tilde{q}_{k}^{l}+\eta \theta_{k}}-\frac{\sum_{i=1}^{N} p_{i}^{l} g_{i, k}^{l}+N_{0}}{g_{k}^{l}}\right]^{+},
\end{gathered}
$$

Where $\delta_{k}, \theta_{k}$ are the lagrange multipliers associated with constraints C3 and C4. Meanwhile, in order to obtain the optimal channel selection, we take the derivative of the subproblem w.r.t $s_{i}^{l}$, which yields to

$$
\frac{\partial \mathcal{L}_{E E}\left(p_{i}^{l}, \alpha_{i}, \beta_{i}\right)}{\partial s_{i}^{l}}=Q_{i}^{l}
$$

whose value is calculated for each channel selection.

$$
s_{i}^{l}=\left\{\begin{array}{lc}
1, & \text { if } Q_{i}^{l}>0 \\
0, & \text { otherwise }
\end{array}\right.
$$

By combining the gradient updates and the channel selection criterion, more than one channel selection can be selected eventually for D2D UEs.

\section{SIMULATION}

In this section, the proposed algorithm is verified through computer simulations. Before the simulation, we define the average energy efficiency as the ratio of the total energy efficiency to the number of channels and simulations. The values of simulation parameters are based on $[11,16]$, and are summarized in Table 1. Fig. 2 shows the locations of

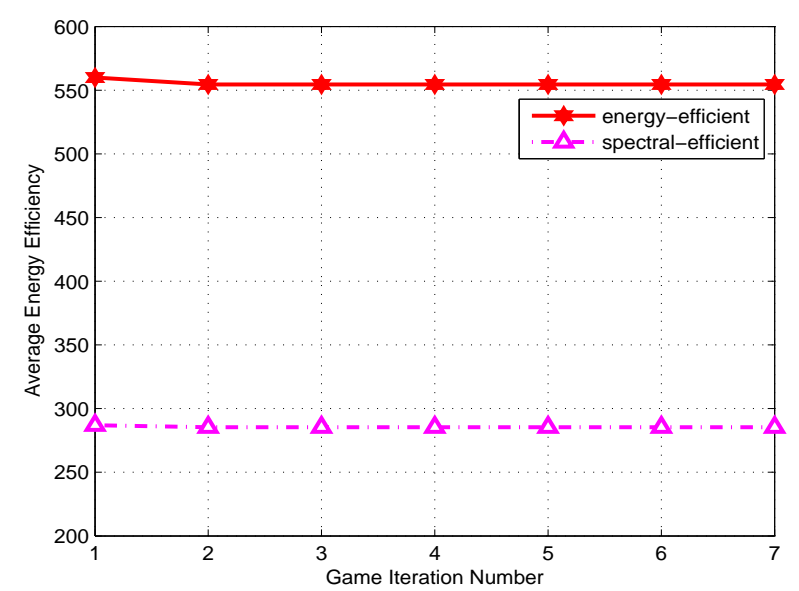

Figure 3: The average energy efficiency of D2D links corresponding to the number of game iterations $\left(N=3, K=3, M=64, p_{i, \max }^{l}=\tilde{p}_{k, \max }^{l}=200\right.$ $\mathbf{m W}, R_{k, \min }^{l}=0.1 \mathrm{bit} / \mathrm{s} / \mathbf{H z}, R_{i, \min }^{l}=0.5 \mathrm{bit} / \mathrm{s} / \mathbf{H z}$, 1000 simulations).

40 D2D UEs (20 D2D pairs) and 10 cellular UEs generated in one simulation. For each simulation, the locations of the cellular UEs and D2D UEs are generated randomly within a cell with a radius of $500 \mathrm{~m}$. The maximum distance between any two D2D UEs that form a D2D pair is $25 \mathrm{~m}$. The channel gain between the transmitter $i$ and the receiver $j$ is calculated as $d_{i, j}^{-2}\left|h_{i, j}\right|^{2}$, where $d_{i, j}$ is the distance between the transmitter $i$ and the receiver $j, h_{i, j}$ is the complex Gaussian channel coefficient that satisfies $h_{i, j} \sim \mathcal{C N}(0,1)$. We compare the proposed EE maximization algorithm (labeled as "energy-efficient") with the SE maximization algorithm which is a distributed iterative algorithm for maximizing each UE's SE. (labeled as "spectral-efficient"). The results are averaged through a total number of 1000 simulations.

Fig. 3 shows the average EE of D2D links corresponding to the number of game iterations. The average EE of the proposed energy-efficient algorithm converge to 554.4333, while the spectral-efficient algorithm converge to 285.3647 . The EE performance is improved by more than $94.29 \%$ in an interference-limited environment. The reason is that in the spectral-efficient algorithm, power consumption is completely ignored in the optimization process.

Fig. 4 shows the average EE of cellular links corresponding to the number of game iterations. Comparing Fig. 4 with Fig. 3, we find that the D2D links can achieve a much better EE than the cellular links due to the proximity gain and the channel reuse gain for shorter transmission distance and proper interference management. The proposed algorithm outperforms the spectral-efficient algorithm by more than 90.3\%. Both Fig. 3 and Fig. 4 show that the proposed iterative energy-efficient algorithm converges very fast to the optimum value.

\section{CONCLUSIONS}

In this paper, we proposed an energy-efficient joint power allocation and channel selection exploiting the properties of 


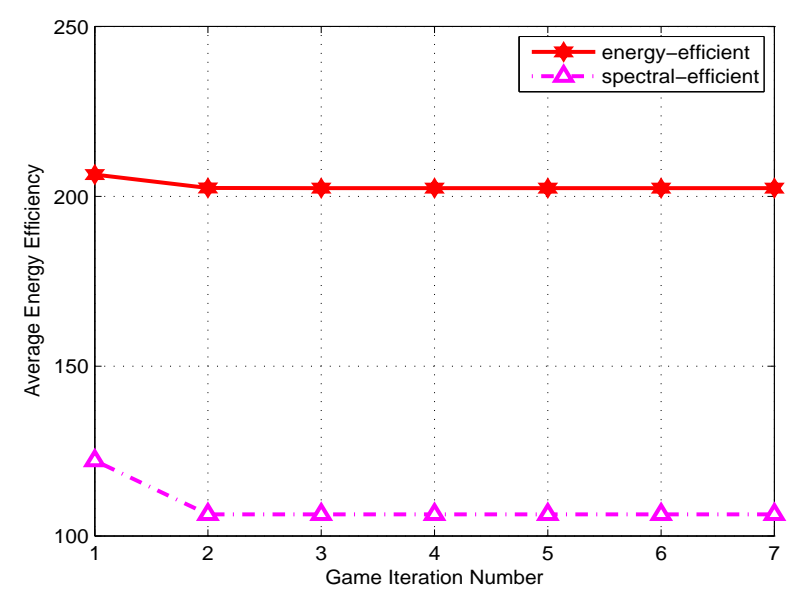

Figure 4: The average energy efficiency of cellular links corresponding to the number of game iterations $\left(N=3, K=3, M=64, p_{i, \max }^{l}=\tilde{p}_{k, \max }^{l}=200\right.$ $\mathbf{m W}, R_{k, \min }^{l}=0.1 \mathrm{bit} / \mathrm{s} / \mathbf{H z}, R_{i, \min }^{l}=0.5 \mathrm{bit} / \mathrm{s} / \mathbf{H z}$, 1000 simulations).

Table 1: Simulation Parameters.

\begin{tabular}{|l|l|}
\hline Parameter & Value \\
\hline Cell radius & $500 \mathrm{~m}$ \\
\hline Maximum D2D distance & $25 \mathrm{~m}$ \\
\hline$p_{i, \max }^{l}, \tilde{p}_{k, \max }^{l}$ & $200 \mathrm{~mW}(23 \mathrm{dBm})$ \\
\hline Constant circuit power $p_{\text {cir }}$ & $10 \mathrm{~mW}(10 \mathrm{dBm})$ \\
\hline Subchannel numbers $M$ & 64 \\
\hline Thermal noise power $N_{0}$ & $10^{-7} \mathrm{~W}$ \\
\hline Number of D2D pairs $N$ & 5 \\
\hline Number of cellular UEs $K$ & 3 \\
\hline PA efficiency $\eta$ & $35 \%$ \\
\hline QoS of cellular UEs $\tilde{R}_{k, \min }^{l}$ & $0.1 \mathrm{bit} / \mathrm{s} / \mathrm{Hz}$ \\
\hline QoS of D2D UEs $R_{i, \min }^{l}$ & $0.5 \mathrm{bit} / \mathrm{s} / \mathrm{Hz}$ \\
\hline
\end{tabular}

the nonlinear fractional programming for D2D UEs and cellular UEs. We derived an iterative algorithm for solving the non-convex problem, which was shown to converge to the optimum value very fast. Simulation results have demonstrated that the proposed EE algorithm can bring significant EE improvement. Compared to the spectral-efficient algorithm, the proposed algorithm improves the EE performance by $94.29 \%$ and $90.3 \%$ for D2D UEs and cellular UEs respectively.

\section{REFERENCES}

[1] L. Wei, R. Hu, Y. Qian, and G. Wu. Enable device-to-device communications underlaying cellular networks:challenges and research aspects. IEEE Trans. Commun., 52(6):90-96, June 2014.

[2] K. Doppler, M. Rinne, C. Wijting, C.Ribeiro, and K. Hugl. Device-to-device communication as an underlay to lte-advanced networks. IEEE Trans. Commun., 47(12):42-49, December 2009.

[3] D. Wu, J. Wang, R. Hu, Y. Cai, and L. Zhou. Energy-efficient resource allocation for device-to-device underlay communication. IEEE Transactions on Wireless Communications, 63(5):2093-2102, June 2014.

[4] K. Doppler, Cąäassio B. Ribeiro, and J. Kneckt. Advances in D2D communications: Energy efficient service and device discovery radio. In 2011 2nd International Conference on Wireless VITAE, (Chennai,February 28-March 3,2011), pages 1-6. IEEE, February-March 2011.

[5] S. Alamouti and R. Sharafat. Resource allocation for energy-efficient device-to-device communication in $4 \mathrm{G}$ networks. In 2014 7th International Symposium on Telecommunications (IST),(Tehran,Septemper 9-11,2014), pages 1058-1063. IEEE, June 2014.

[6] D. Wu, L. Zhou, R. Hu, and Y. Qian. The role of mobility for D2D communications in lte-advanced networks: energy vs. bandwidth efficiency. Wireless Communications, IEEE., 21(2):66-71, April 2014.

[7] S. Mumtaz, K. Mohammed S. Huq, A. Radwan, J. Rodriguez, and R. Aguiar. Energy efficient interference-aware resource allocation in LTE-D2D communication. In 2014 IEEE International Conference on Communications (ICC), (Sydney, NSW, June 10-14,2014), pages 282-287. IEEE, June 2014.

[8] L. Wei, R. Hu, Y. Qian, and G. Wu. Energy-efficiency and spectrum-efficiency of multi-hop device-to-device communications underlaying cellular networks. IEEE Transactions on Vehicular Technology, PP(99):1-13, January 2015.

[9] A. Prasad, A. Kunz, G. Velev, K. Samdanis, and J. Song. Energy-efficient D2D discovery for proximity services in 3GPP LTE-Advanced networks. Vehicular Technology Magazine, IEEE, 9(4):40-50, December 2014.

[10] A. Antonopoulos, E. Kartsakli, and C. Verikoukis. Game theoretic D2D content dissemination in $4 \mathrm{G}$ cellular networks. Communications Magazine, IEEE, 52(6):125-132, June 2014.

[11] Z. Zhou, M. Dong, K. Ota, J. Wu, and T. Sato. Energy efficiency and spectral efficiency tradeoff in device-to-device (D2D) communications. Wireless Communications Letters, IEEE, pages 485-488, October 2014.

[12] Z. Zhou, M. Dong, K. Ota, R. Shi, Z. Liu, and T. Sato. A game-theoretic approach to energy-efficient resource allocation in device-to-device underlay communications. IET Communications, 9(3):375-385, February 2015.

[13] Z. Luo and S. Zhang. Dynamic spectrum management: Complexity and duality. IEEE journal of selected topics in signal processing, 2(1):57-73, February 2008.

[14] W. Dinkelbach. On nonlinear fractional programming. Management Science, 13(7):492-498, March 1967.

[15] S. Boyd and L. Vandenberghe. Convex Optimization. Cambridge University Press, Cambridge, UK, 2004.

[16] C. Xu, L. Song, Z. Han, and Q. Zhao. Efficiency resource allocation for device-to-device underlay communication systems: a reverse iterative combinatorial auction based approach. IEEE J. Sel. Areas Commun., 31(9):348-358, September 2013. 